\title{
Global miRNA expression and correlation with mRNA levels in primary human bone cells
}

\author{
NAVYA LAXMAN, ${ }^{1,2}$ CARL-JOHAN RUBIN, ${ }^{3}$ HANS MALLMIN, ${ }^{4}$ OLLE NILSSON, ${ }^{4}$ TOMI PASTINEN,,$^{5,6}$ \\ ELIN GRUNDBERG, ${ }^{5,6}$ and ANDREAS KINDMARK ${ }^{1,2}$ \\ ${ }^{1}$ Department of Medical Sciences, Uppsala University, SE-75185 Uppsala, Sweden \\ ${ }^{2}$ Science for Life Laboratory, Department of Medical Sciences, Uppsala University Hospital, SE-75185 Uppsala, Sweden \\ ${ }^{3}$ Department of Medical Biochemistry and Microbiology, Uppsala University, SE-75185 Uppsala, Sweden \\ ${ }^{4}$ Department of Surgical Sciences, Uppsala University, SE-75185 Uppsala, Sweden \\ ${ }^{5}$ Department of Human Genetics, McGill University, Montreal, Quebec, Canada H3A 1B1 \\ ${ }^{6}$ Genome Quebec Innovation Centre, McGill University, Montreal, Quebec, Canada H3A 0G1
}

\begin{abstract}
MicroRNAs (miRNAs) are important post-transcriptional regulators that have recently introduced an additional level of intricacy to our understanding of gene regulation. The aim of this study was to investigate miRNA-mRNA interactions that may be relevant for bone metabolism by assessing correlations and interindividual variability in miRNA levels as well as global correlations between miRNA and mRNA levels in a large cohort of primary human osteoblasts (HOBs) obtained during orthopedic surgery in otherwise healthy individuals. We identified differential expression (DE) of 24 miRNAs, and found 9 miRNAs exhibiting DE between males and females. We identified hsa-miR-29b, hsa-miR-30c2, and hsa-miR-125b and their target genes as important modulators of bone metabolism. Further, we used an integrated analysis of global miRNA-mRNA correlations, mRNAexpression profiling, DE, bioinformatics analysis, and functional studies to identify novel target genes for miRNAs with the potential to regulate osteoblast differentiation and extracellular matrix production. Functional studies by overexpression and knockdown of miRNAs showed that, the differentially expressed miRNAs hsa-miR-29b, hsa-miR-30c2, and hsa-miR-125b target genes highly relevant to bone metabolism, e.g., collagen, type I, $\alpha 1$ (COL1A1), osteonectin (SPARC), Runt-related transcription factor $2(R U N X 2)$, osteocalcin (BGLAP), and frizzled-related protein (FRZB). These miRNAs orchestrate the activities of key regulators of osteoblast differentiation and extracellular matrix proteins by their convergent action on target genes and pathways to control the skeletal gene expression.
\end{abstract}

Keywords: miRNA; mRNA; osteoblasts; interindividual variation; differential expression

\section{INTRODUCTION}

MicroRNAs (miRNAs) are small ( 22 nt long), singlestranded noncoding RNAs which regulate gene expression primarily by binding to partially complementary sites in the $3^{\prime}$ UTR of mRNAs and inhibit gene expression either by facilitating mRNA degradation or by inhibition of translation (Bartel 2004; Doench and Sharp 2004; Engels and Hutvagner 2006; Rajewsky 2006; Hu and Coller 2012; Wilczynska and Bushell 2015). Recent evidence from Bartel et al., however, suggests that the predominant mechanism by which miRNAs regulate protein levels of target genes is mRNA stability rather than on translation (Guo et al. 2010). Recent studies indicate that over one-third of all human genes are regulated by microRNAs (Davis and Hata 2009). Studies suggest that a single gene can be targeted by a cluster of miRNAs,

Corresponding author: navya.laxman@medsci.uu.se

Article published online ahead of print. Article and publication date are at http://www.rnajournal.org/cgi/doi/10.1261/rna.049148.114. Freely available online through the RNA Open Access option. but also that a single miRNA can target many protein coding genes. As the field of miRNA studies has evolved, the actions of miRNAs have been shown to be surprisingly complex, and intricate networks of factors stringently control miRNA processing and biological activities.

Osteoporosis is a common disease, characterized by diminished bone mineral density (BMD) and bone strength and consequently an increased risk for fractures. The genetic contribution to osteoporosis is well known and much effort has been made to identify genetic variants associated with the susceptibility to osteoporotic fractures; both for the discovery of novel drug targets but also for the identification of risk factors.

Expression of different subsets of bone-regulating miRNAs (with the proposed name osteomiRs) during different stages of osteoblast maturation and osteoclastogenesis have been

(c) 2015 Laxman et al. This article, published in $R N A$, is available under a Creative Commons License (Attribution-NonCommercial 4.0 International), as described at http://creativecommons.org/licenses/by-nc/4.0/. 
reported, regulating the timing and progression of cellular differentiation programs (Lian et al. 2012). The miRNA families; hsa-miR-29, hsa-miR-let-7, and hsa-miR-26 influence bone formation by targeting a number of collagens and other extracellular matrix proteins (Zhao et al. 2014). It has been shown that hsa-miR-125b down-regulates cell proliferation by inhibiting osteoblast differentiation (Miyazono et al. 2005). Hsa-miR-29b is a positive regulator of osteoblast differentiation, targeting bone matrix RNAs, including COL1A1 (Kapinas et al. 2010) and COL3A1, down-regulating inhibitors such as histone deacetylase (HDAC) 4, TGF 33 , $A c v R 2 A$, and CTNNBIP1 (catenin $\beta$-interacting protein I) in rat and mouse cells (Li et al. 2009). Studies on human mesenchymal stem cells (MSC) revealed that hsa-miR-31 negatively regulates Osterix expression, which in turn targets BGLAP and COL1A1 genes during early osteoblastic differentiation, thereby making hsa-miR-31 a potentially important regulator of bone mineralization (Baglì et al. 2013). Recent studies identified nine up-regulated miRNAs in osteoporotic patients, suggesting that alterations in the levels of circulating miRNAs in serum is associated with either increased osteoclastogenesis or inhibited osteoblast differentiation, and that circulating miRNA levels could be used as novel biomarkers for diagnostic purposes (Seeliger et al. 2014).

\section{RESULTS}

\section{Global mRNA expression}

The global mRNA-expression profiles from primary human osteoblasts (HOBs) from 95 individuals were obtained using the Illumina HumRef-8v2 arrays. Microarray data have been deposited in the Gene Expression Omnibus (GEO) (www. ncbi.nlm.nih.gov/geo, accession no. GSE15678).

\section{microRNA profiling in human bone samples by microarray}

Normalization and data filtering of the 757 probes resulted in 251 high quality probe signals passing predetermined quality criteria, and these were used in downstream analyses (Supplemental Table 1a). In the initial analysis of data, we determined relative levels of miRNA expression and differentially expressed (DE) miRNAs, defined as having $M$-values with $\mathrm{SD}>0.5$, resulting in 24 DE miRNAs overall (Fig. 1A; Supplemental Table 1a). When assessing gender differences, 9 of 251 miRNA had significantly different expression levels between males and females in two-tailed $t$-test with a $P$-value cut-off at $<0.05$ (Fig. 1B; Supplemental Table 1b). The gender difference is further exemplified in Supplemental Figure 2 using principal component analysis (PCA) showing separation of female and male clusters. The annotation miRPlus in tables and figures refers to Exiqon in-licensed human sequences not yet annotated in miRBase (latest release version 21: June 2014).

\section{microRNA profiling in human bone samples by real-time RT-PCR}

From the 251 expressed miRNAs identified from the LNA microarray experiment a subset of miRNAs with readily available assays for verification was chosen. The miRNAs were selected based on the LNA array data with either DE between genders with a $P<0.05$ (hsa-miR-29b, hsa-miR-99a, and hsamiR-140-3p), and/or overall DE with an SD > 0.5 (hsa-miR30c2, hsa-miR-503, hsa-miR-31, hsa-miR-335, hsa-miR-22, and hsa-miR-198), or based on high Hy3/Hy5 average intensity on the LNA array (hsa-miR-125b, data not shown), and for use as controls (hsa-miR-191 and hsa-let-7a, see below). The expression profiles of these 12 miRNAs were successfully quantitated in HOBs from 95 individuals using TaqMan MicroRNA Assays. There was no correlation between miRNA levels and age of the bone cell donor $(P=0.23-$ 0.97 , bivariate analysis by age). The miRNAs exhibited some degree of correlation overall, less pronounced for hsa-miR335, hsa-miR-198, and hsa-miR-29b. The correlation pattern differed slightly between males and females (Supplemental Table 2). The validation by TaqMan analyses showed that 9/ 10 miRNAs (hsa-miR-125b, hsa-miR-29b, hsa-miR-30c2, hsa-miR-31, hsa-miR-99a, hsa-miR-140-3p, hsa-miR-198, hsa-miR-335, and hsa-miR-503) exhibited DE with overall SD 0.9-17.2. Four out of 10 tested miRNAs showed the same trend for differences in expression between males and females as was previously observed in the microarray analysis; hsa-miR-125b $(P=0.089)$, hsa-miR-29b $(P=0.113)$, hsamiR-198 $(P=0.064)$, with hsa-miR-31 $(P=0.049)$ reaching statistical significance (Fig. 2B).

\section{Correlations between miRNA- and mRNA levels}

Analysis revealed large numbers of significant correlations at the $P<0.05$ level with preponderance for significant correlations in females (Fig. 2A). Using an FDR cut-off of 0.05, the number of remaining correlations was reduced (Table 1 ), but the pattern of more correlations being observed for females remained. The number of positive correlations was generally higher than negative correlations, with a mean ratio for positive versus negative correlations of 1.5 (range 0.9-2.7). hsa-miR-29b showed the highest number of significant correlations followed by an intermediate group consisting of hsa-miR-335, hsa-miR-140-3p, hsa-miR-99a, hsa-miR-31, hsa-miR-22, and hsa-miR-125.

Quantile-quantile (Q-Q) plots performed on the 10 tested miRNAs in the overall data set showed substantial egression of the observed findings from the null hypothesis of no correlation, indicative of significant correlations (Supplemental Fig. 3 ). In addition, an overrepresentation analysis showed that mRNAs having a predicted hsa-miR-29b target site in their 3' UTR were fourfold enriched among those mRNA most significantly correlated with miR-29b (FDR <0.01) (Supplemental Fig. 4). This further strengthens the case that the significant correlations we observed for hsa-miR-29b are indeed true primary effects due to miRNA-mRNA interactions. 

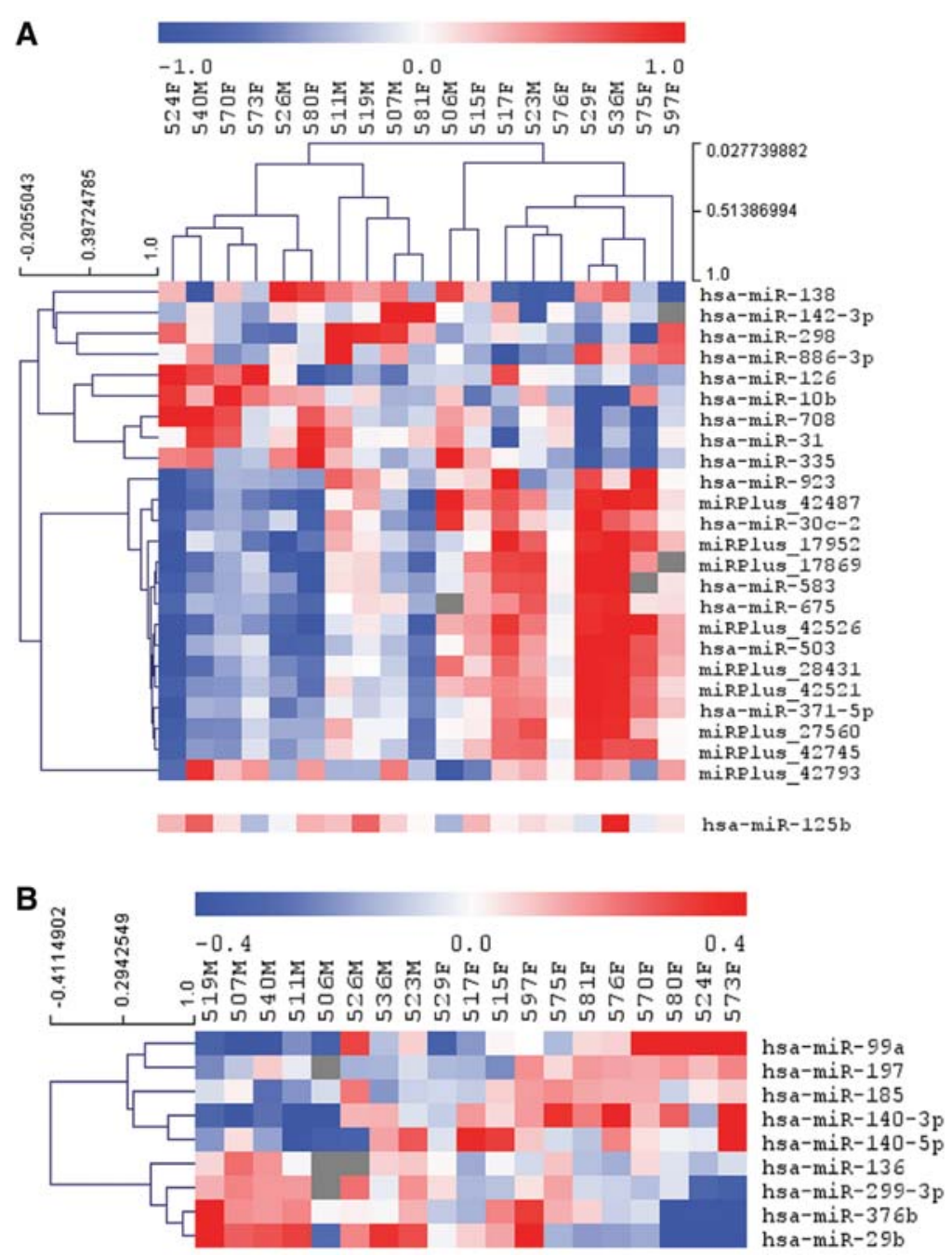

FIGURE 1. Heatmaps representing miRNA expression. (A) Heatmap of miRNA expression by LNA microarray in human osteoblasts from eight males and 11 females. Out of the 251 expressed miRNAs, a heatmap was generated for the 24 miRNAs differentially expressed by $\log _{2}$ (Hy3/Hy5) ratios with a variation across all samples having $\mathrm{SD}>0.50$. For comparison, expression levels for hsa-miR-125b are shown below the 24 DE miRNAs. (B) Heatmap depicting variation in miRNA expression between females and males. Out of the expressed miRNA, nine exhibited differential expression levels between males and females based on $\log _{2}(\mathrm{Hy} 3 / \mathrm{Hy} 5)$ ratios significantly different between males and females using a two-tailed $t$-test and $P$-values $<0.05$. Clustering was made and heatmaps were processed and displayed using MultiExperiment Viewer.

A number of genes involved in bone metabolism exhibited a statistically significantly correlation between miRNA and mRNA expression. COL1A1 $(P=0.0001)$ and SPARC $(P=$ 0.001 ) showed a negative correlation to hsa-miR-29b (Fig. 3B), RUNX2 $(P=0.0148), F R Z B(P=0.003)$, and BGLAP $(P=0.0325)$ were negatively correlated with hsa-miR-30c2 (Fig. 4B) and FRZB $(P=0.0117)$ and BGLAP $(P=0.0450)$ were negatively correlated with hsa-miR-125b (Fig. 5B).

\section{Bioinformatic pathway analysis}

The miRNA versus mRNA correlation data set was interrogated by the Ingenuity Pathway Analysis software (IPA, Ingenuity
Systems). Analyses were performed for the overall data set, and for male and females assessed separately. IPA analysis on the miRNA versus mRNA correlation data set yielded several pathways with known or putative relations to bone metabolism reaching a $P$-value $<0.05$. Although the majority of the significant pathways were unique for each miRNA, one pathway reaching statistical significance was the Wnt/ $\beta$-catenin signaling pathway, for hsa-miR-29b, hsa-miR125b, and hsa-miR-30c2 (Supplemental Fig. 5 for hsa-miR-29b).

\section{hsa-miR-29b, hsa-miR-30c2, and hsa- miR-125b regulate osteogenesis}

Functional studies of hsa-miR-29b, hsamiR-30c2, and hsa-miR-125b were performed by overexpression and knockdown studies in HOBs. The putative binding sites in the $3^{\prime}$ UTRs of hsa-miR29b in COL1A1 and SPARC mRNA (Fig. 3A), of hsa-miR-30c2 RUNX2, FRZB, and BGLAP (Fig. 4A), of hsamiR-125b FRZB and BGLAP (Fig. 5A), were predicted by the softwares TargetScan, miRanda, and PicTar. The overexpression studies show that augmented amount of hsa-miR-29b repressed CollA1 and SPARC, whereas hsa-mir29b inhibitor increased CollA1 and SPARC at the mRNA and protein level. Augmented amounts of hsa-miR-30c2 repressed RUNX2, FRZB, and BGLAP, whereas hsa-mir-30c2 inhibitor elevated the amount of RUNX2, FRZB, and $B G L A P$. Augmented amounts of hsamiR-125b repressed FRZB and BGLAP, whereas hsa-miR-125b inhibitor elevated the amount of FRZB and BGLAP as assessed by RT-PCR (Figs. 3-5C) and Western blotting (Figs. 3-5D). In vitro matrix mineralization levels was visualized and quantified by ALP and Alizarin red staining, showing increased mineralization with hsa-miR-29b, hsa-miR-30c2, and hsa-miR-125b inhibitors and a decreased mineralization by hsa-miR-29b, hsa-miR-30c2, and hsa-miR-mimics (Figs. 3-5E,F).

\section{DISCUSSION}

The human genome is estimated to encode $>1000$ miRNAs which may regulate up to $\sim 60 \%$ of human genes (Lian et al. 2012). In recent years, a number of studies have identified 
A

B

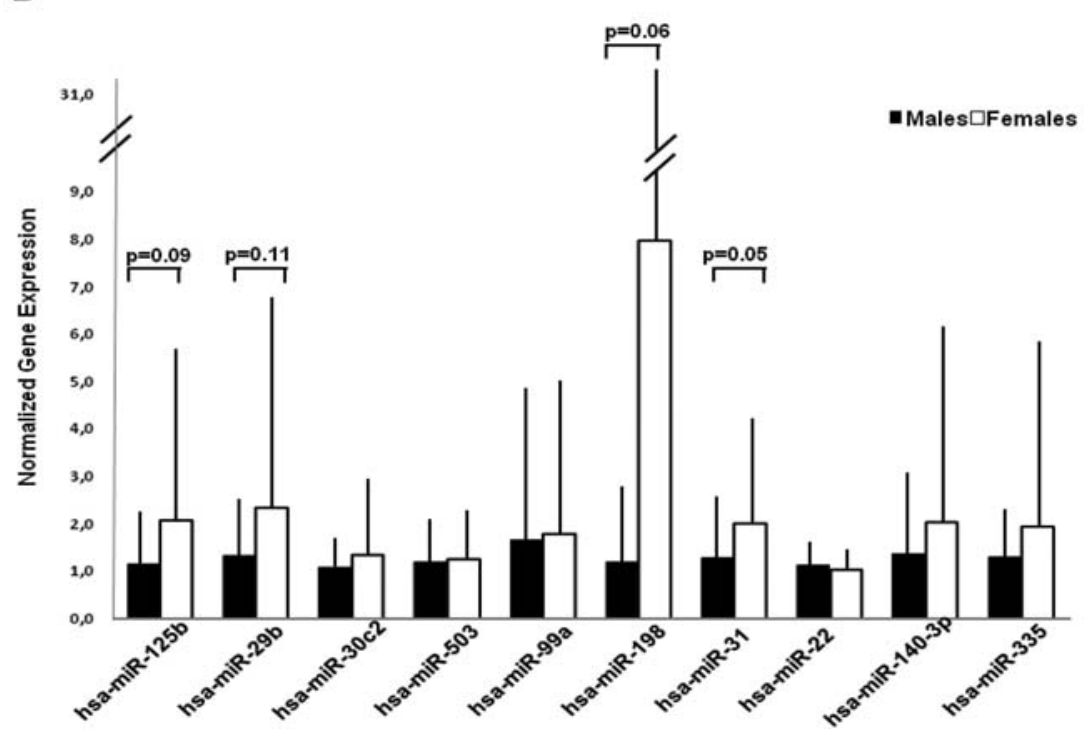

FIGURE 2. miRNA-expression profiles showing segregation between females and males. $(A)$ Venn diagram depicting the number of correlations miRNA versus mRNA with $P<0.05$, and the number of shared correlations between males, females, and the overall data set. $(B)$ TaqMan results for the subset selected for verification of miRNA LNA array data. The selection was based on overall differential expression and/or differential expression between males and females. Bars represent means with SD. $P$-values for $T$-tests for independent samples of males versus females are indicated above the respective bars.

unique miRNA signatures for several diseases (Philippidou et al. 2010). Advances in miRNA basic research, discovery of new miRNAs and their target networks have been paralleled by developments and improvements in miRNA detection techniques. In our study, we have used sensitive LNA miRNA microarrays and specific qPCR protocols to assess the miRNAome in primary human bone cells.

To explore whether microRNAs have a role in human bone dynamics it is necessary to determine which miRNAs are active within bone. So far, the function of a number of miRNAs has been characterized in bone; e.g., hsa-miR125b, hsa-miR-29b, and hsa-miR-30a have been associated with osteoblast differentiation in human multipotent mesenchymal stromal cells (MSCs) (Gao et al. 2011). In the present study, the miRNA-expression profile of primary human bone cells was analyzed, revealing expression of $\sim 250$ miRNAs in HOBs. We then further validated differential expression of a subset of 10 miRNAs in 95 individuals by qPCR, and correlated the miRNA levels with global mRNA levels in these same individuals and found that hsamiR-29b had the largest number of significant correlations to mRNA levels.

Validation of miRNA targets is an ongoing process, and the TarBase v6.0 database (Vergoulis et al. 2012) currently contains $\sim 65,000$ experimentally validated miRNA-gene interactions. A number of these validated targets are present in our data set. The predicted targets of the miRNAs in this study were also verified using the softwares TargetScan, miRanda, and PicTar. hsa-mir-29b, hsa-mir-30c2, and hsa-mir-125b were differentially expressed in our data set and for these miRNAs, our miRNAmRNA correlation studies show a number of statistically significant correlations for genes involved in bone metabolism, giving these three miRNAs high priority for assessing their effects on osteoblast differentiation and mineralization.

hsa-miR-29b has been shown to target the most abundant collagenous matrix gene COL1A1 (Li et al. 2009), and hsamiR-29a and $-29 c$ to target the most abundant noncollagenous matrix gene SPARC (Kapinas et al. 2009). Our data now show a novel negative correlation between hsa-miR-29b and SPARC mRNA levels, a finding which we then further confirmed by functional studies at the protein level. Synthesis of collagenous and noncollagenous proteins is crucial for the formation of extracellular matrix by the osteoblast. hsa-miR-29b suppresses the expression of collagen proteins allowing the collagen fibril matrix to mature for mineral deposition (Fig. 6). Previous studies have shown that collagen proteins are not suppressed in immature osteoblasts but attenuated only in mature osteoblasts (Li et al. 2009). As osteoblasts differentiate, the expression and synthesis of extracellular matrix genes are reduced (Kalajzic et al. 2005). In our functional studies on $\mathrm{HOBs}$ and matrix proteins, we confirm that COL1A1 shows a significant negative correlation and also show a novel negative correlation between and SPARC and hsa-miR-29b both on the mRNA and protein level.

RUNX2 is a key regulator of osteogenesis and it is subjected to multiple levels of regulation of miRNAs. During early stages of osteoblast differentiation, RUNX2 is up-regulated, activating major matrix genes, while RUNX2 is down-regulated in mature osteoblasts (Fig. 6). It is well established that hsa-miR-30c mediates inhibition of osteoblastic differentiation by targeting RUNX2 (Zhang et al. 2011). In our 
TABLE 1. miRNA:mRNA correlations

\begin{tabular}{|c|c|c|c|c|c|c|c|}
\hline \multirow[b]{2}{*}{ miRNA } & \multicolumn{2}{|c|}{ Total number of correlations } & \multicolumn{2}{|c|}{ Positive correlations } & \multicolumn{2}{|c|}{ Negative correlations } & \multirow{2}{*}{$\begin{array}{c}P \text {-value range } \\
(\text { for FDR }<0.051)\end{array}$} \\
\hline & $P$-value $<0.05$ & FDR $<0.05$ & $P$-value $<0.05$ & FDR $<0.05$ & $P$-value $<0.05$ & FDR $<0.05$ & \\
\hline \multicolumn{8}{|l|}{ hsa-miR-125b } \\
\hline Female & 2294 & 54 & 1186 & 50 & 1108 & 4 & $2.2 \times 10^{-9}-1.1 \times 10^{-4}$ \\
\hline Male & 739 & 0 & 427 & 0 & 312 & 0 & NA \\
\hline Combined & 942 & 5 & 555 & 5 & 387 & 0 & $7.3 \times 10^{-8}-4.6 \times 10^{-6}$ \\
\hline \multicolumn{8}{|l|}{ hsa-miR-22 } \\
\hline Female & 705 & 0 & 333 & 0 & 372 & 0 & NA \\
\hline Male & 1206 & 9 & 869 & 9 & 337 & 0 & $1.6 \times 10^{-6}-1.8 \times 10^{-5}$ \\
\hline Combined & 1572 & 37 & 1099 & 36 & 473 & 1 & $8.6 \times 10^{-10}-1.1 \times 10^{-5}$ \\
\hline \multicolumn{8}{|l|}{ hsa-miR-29b } \\
\hline Female & 3780 & 501 & 1908 & 258 & 1872 & 243 & $7.1 \times 10^{-8}-1.5 \times 10^{-3}$ \\
\hline Male & 3355 & 0 & 1744 & 0 & 1611 & 0 & NA \\
\hline Combined & 4915 & 2018 & 2491 & 1018 & 2424 & 1000 & $1.6 \times 10^{-10}-6.1 \times 10^{-3}$ \\
\hline \multicolumn{8}{|l|}{ hsa-miR-30c2 } \\
\hline Female & 2378 & 0 & 1290 & 0 & 1088 & 0 & NA \\
\hline Male & 827 & 0 & 422 & 0 & 405 & 0 & NA \\
\hline Combined & 2506 & 2 & 1431 & 1 & 1075 & 1 & $5.4 \times 10^{-6}-4.5 \times 10^{-6}$ \\
\hline \multicolumn{8}{|l|}{ hsa-miR-31 } \\
\hline Female & 1661 & 1 & 822 & 1 & 839 & 0 & $1.1 \times 10^{-6}$ \\
\hline Male & 2264 & 18 & 1198 & 15 & 1066 & 3 & $1.2 \times 10^{-7}-5.2 \times 10^{-5}$ \\
\hline Combined & 2567 & 0 & 1231 & 0 & 1336 & 0 & NA \\
\hline \multicolumn{8}{|l|}{ hsa-miR-99a } \\
\hline Female & 1449 & 8 & 812 & 8 & 637 & 0 & $2.2 \times 10^{-8}-1.6 \times 10^{-5}$ \\
\hline Male & 1280 & 15 & 922 & 13 & 358 & 2 & $2.2 \times 10^{-9}-3.6 \times 10^{-5}$ \\
\hline Combined & 1058 & 5 & 744 & 5 & 314 & 0 & $4.4 \times 10^{-8}-2.0 \times 10^{-6}$ \\
\hline \multicolumn{8}{|c|}{ hsa-miR-140-3p } \\
\hline Female & 2158 & 29 & 1150 & 28 & 1008 & 1 & $2.4 \times 10^{-10}-7.2 \times 10^{-5}$ \\
\hline Male & 514 & 1 & 376 & 1 & 138 & 0 & $2.9 \times 10^{-9}$ \\
\hline Combined & 658 & 1 & 438 & 1 & 220 & 0 & $3.4 \times 10^{-9}$ \\
\hline \multicolumn{8}{|l|}{ hsa-miR-198 } \\
\hline Female & 404 & 0 & 238 & 0 & 166 & 0 & NA \\
\hline Male & 660 & 0 & 441 & 0 & 219 & 0 & NA \\
\hline Combined & 444 & 0 & 283 & 0 & 161 & 0 & NA \\
\hline \multicolumn{8}{|l|}{ hsa-miR-335 } \\
\hline Female & 1475 & 70 & 851 & 64 & 624 & 6 & $5.5 \times 10^{-12}-2.1 \times 10^{-4}$ \\
\hline Male & 1351 & 8 & 775 & 7 & 576 & 1 & $8.5 \times 10^{-8}-2.4 \times 10^{-5}$ \\
\hline Combined & 1125 & 12 & 676 & 11 & 449 & 1 & $1.4 \times 10^{-7}-2.7 \times 10^{-5}$ \\
\hline \multicolumn{8}{|l|}{ hsa-miR-503 } \\
\hline Female & 2118 & 0 & 977 & 0 & 1141 & 0 & NA \\
\hline Male & 486 & 0 & 298 & 0 & 188 & 0 & NA \\
\hline Combined & 1591 & 0 & 777 & 0 & 814 & 0 & NA \\
\hline
\end{tabular}

Correlation between miRNA fold-change values and global mRNA-expression levels in HOBs from 95 individuals. This table shows an overview of the number of correlations distributed between males, females, and the combined data set. Positive and negative correlations are indicated in their respective columns. Statistical analysis identified large number of significant correlations at the $P<0.05$ level. Using the more stringent FDR cut-off of 0.05 , the number of remaining correlations was significantly reduced. hsa-miR-29b showed the highest number of significant correlations followed by the intermediate group of hsa-miR-335, hsa-miR-140-3p, hsa-miR-99a, hsa-miR-31, hsa-miR-22, and hsa-miR-125.

functional studies on HOBs, we confirm a significant negative effect of hsa-miR-30c2 on RUNX2. In addition, our results also show a novel effect of hsa-miR-30c2 and hsamiR-125b with negative correlations to expression levels of $F R Z B$ and the late-stage osteoblast marker BGLAP. Our pathway analysis showed many statistically significant pathways that were unique for each miRNA, while one pathway that reached statistical significance shared by many miRNA was the $\mathrm{Wnt} / \beta$-catenin signaling pathway. MiRNAs modulate Wnt signaling by targeting and suppressing negative regula- tors of the Wnt pathway, e.g., FRZB/sFRPs. Our functional results show that hsa-miR-30c2 and hsa-miR-125b are negatively correlated to $F R Z B / s F R P s$ indicating an activating effect on the $\mathrm{Wnt} / \beta$-catenin pathway. Furthermore, our results support previous findings that hsa-miR-29b is a key downregulator of Wnt antagonists like Dkk1, Kremen2, sFRP2, and WIF1 (Zhou et al. 2011). The data also show a positive correlation of hsa-miR-29b with Wnt1, one of the activators of canonical Wnt signaling pathway and $\beta$-catenin which activates target gene transcription ( $\mathrm{Li}$ et al. 2011). 
A

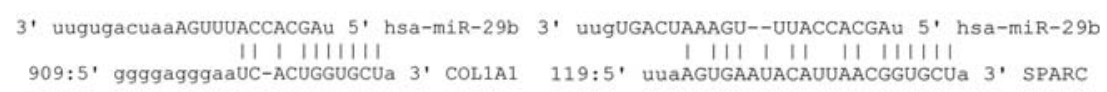
909:5' ggggagggaauC-ACUGGUGCUa $3^{\prime}$ COL1A1 $119: 5^{\prime}$ uuaAgugAaUACAUUAACGGUGCUa $3^{\prime}$ SPARC

B

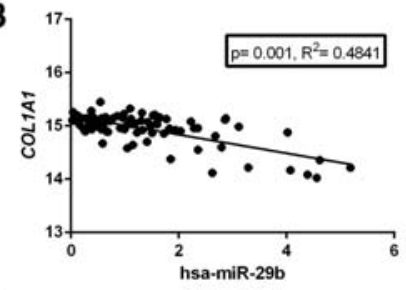

C

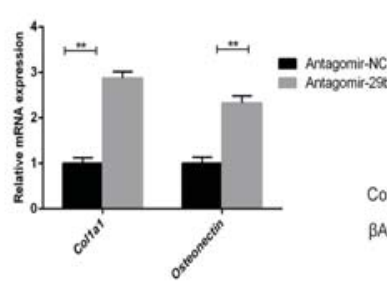

D

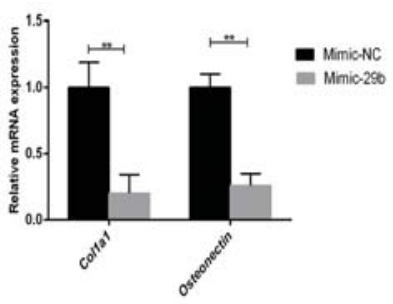

$\mathbf{F}$
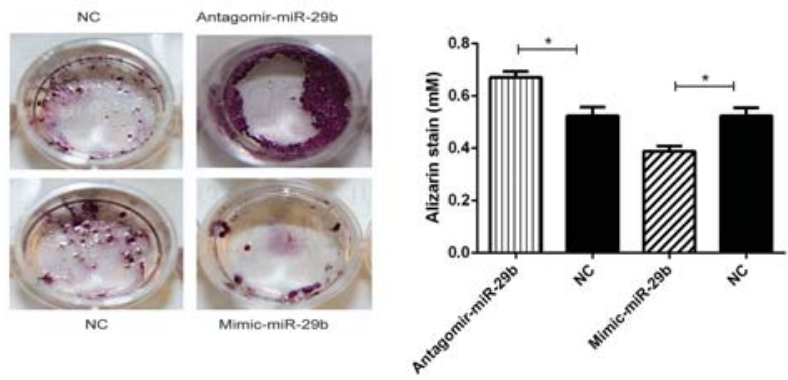

FIGURE 3. hsa-miR-29b negatively regulates CollA1 and SPARC in primary human bone cells. (A) Predicted binding sites for miR-29b in CollA1 and SPARC $3^{\prime}$ UTR by the TargetScan, miRanda, and PicTar softwares. $(B)$ Pearson correlation scatter plots showing a negative correlation between hsa-miR-29b and COL1A1 $(P=0.001)$ and with SPARC $(P=0.001)$. (C) RT-PCR analysis of COL1A1 and SPARC mRNA levels normalized to GAPDH. (D) The amount of COL1A1 protein was assessed by Western blot analysis with $\beta$-actin as a loading control (left panel) and SPARC protein secreted into conditioned media was quantified by Western blot. Mean \pm SD * is significantly different from negative control (NC) (right panel). (E) ALP staining was visualized at $10 \times$ magnification. $(F)$ Deposition of calcium was visualized (left panel) and quantified (right panel), by Alizarin red stain in HOBs after they were treated in osteogenic medium but for $12 \mathrm{~d}$.

Compared with the paradigm that miRNAs are repressive in nature, our miRNA versus mRNA correlation data showed a higher number of significant positive correlations than negative correlations, which is intriguing. Though unexpected, the detection of positive correlations between miRNA and miRNA has been reported in many studies (Vasudevan et al. 2007; Nunez-Iglesias et al. 2010). Gene regulatory networks often include feedback motifs, and miRNAs are interwoven into such complex regulatory networks which coordinate transcriptional regulation in signaling networks (Inui et al. 2010). The overrepresentation of positive correlations in our data could be due to downstream effects, perhaps due to inhibited repression or perturbations of feedback loops (Martinez et al. 2008; Sun et al. 2012).

The aim of this study was to identify miRNAs involved in bone metabolism, using primary human osteoblasts (HOBs). By using human primary cells rather than rat or mouse, or rodent cell lines, we hope to circumvent issues regarding species or strain-specific effects. Our study is unique in demonstrating that expression of miRNAs exhibits interindividual variation in primary bone cells from a large cohort and also in showing differential expression of miRNAs between males and females, which has not been shown before in studies of bone cells. Although the initial findings regarding gender differences found in 20 subjects by LNA array showed the same trend when analyzing by TaqMan in HOBs from 95 subjects, only miR-31 remained significant. The difference in miRNA levels between cells from males and females was observed in culture conditions that were identical for all cells, which indicates either an underlying gender difference, or that cells from males and females have been primed epigenetically or by other means to express miRNAs differently in response to changes in hormonal milieu. These findings are intriguing, and although our data were obtained by two different quantification techniques, findings need to be replicated in larger experimental settings for putative gender differences to be further elucidated.

In conclusion, this study shows that several miRNAs exhibiting interindividual variation act in important metabolic pathways, with hsa-miR-29b, hsa-miR-30c2, and hsa-miR$125 \mathrm{~b}$ identified as affecting osteoblast differentiation and mineralization by targeting genes highly relevant to bone metabolism. These miRNAs play a regulatory role affecting osteoblast differentiation and extracellular matrix production. In this study, we used an integrated analysis of global mRNA-miRNA correlations, bioinformatics analysis, and functional studies to identify novel target genes for miRNAs with the potential to regulate osteoblast differentiation. 
A

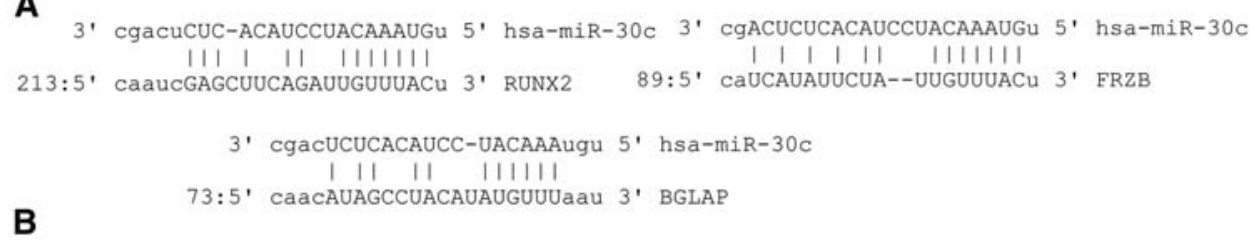

B

73:5' caacAUAgCCUACAUAUgUUUaau $3^{\prime}$ BgLAP
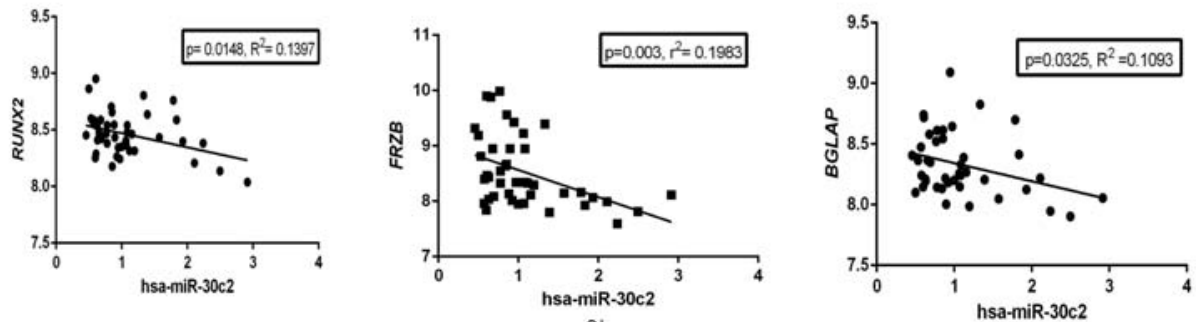

C
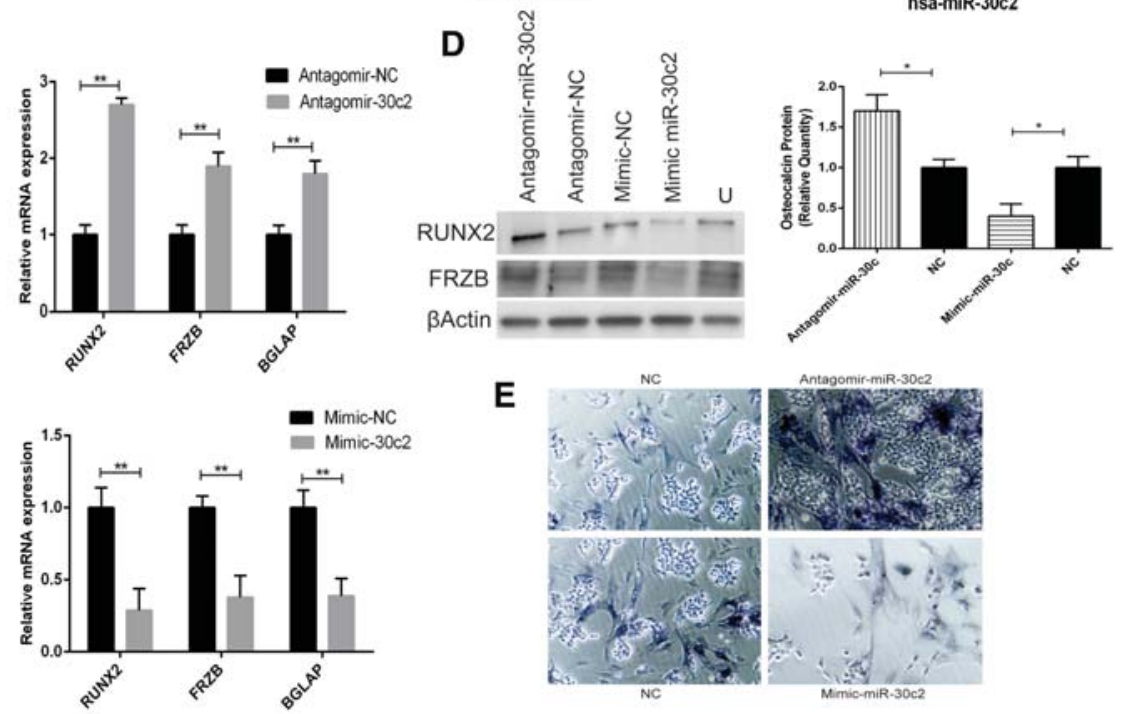

E

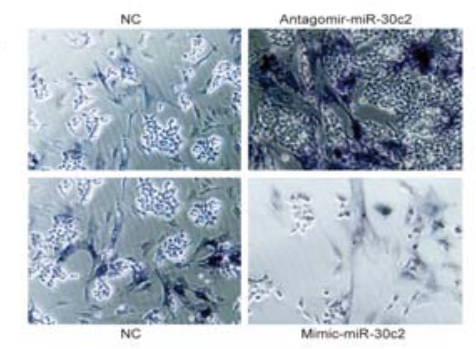

$\mathbf{F}$
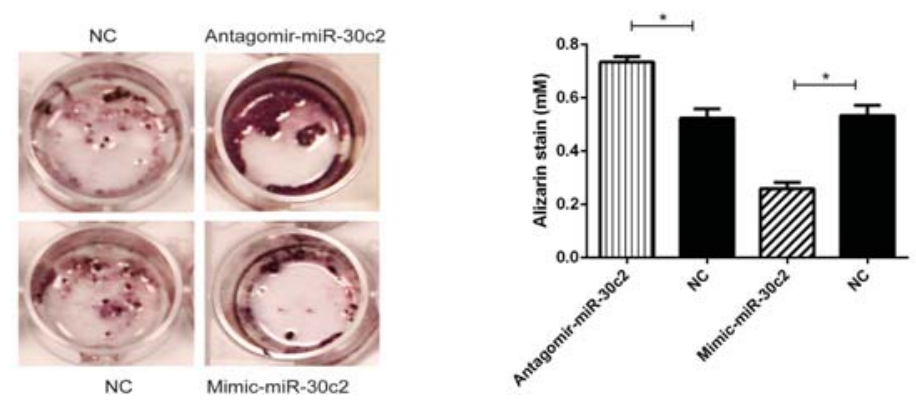

FIGURE 4. hsa-miR-30c2 negatively regulates RUNX2, FRZB, and BGLAP in primary human bone cells. (A) Predicted binding sites for hsa-miR$30 \mathrm{c} 2$ in $R U N X 2, F R Z B$, and BGLAP $3^{\prime}$ UTR by the TargetScan, miRanda, and PicTar softwares. (B) Pearson correlation scatter plots showing a negative correlation between hsa-miR-30c2 RUNX2 $(P=0.0148), F R Z B(P=0.003)$, and BGLAP $(P=0.0325)$. (C) RT-PCR analysis of RUNX2, FRZB, and $B G L A P$ mRNA levels normalized to GAPDH. $(D)$ The amount of RUNX2 and FRZB was assessed by Western blot analysis with $\beta$-actin as a loading control (left panel) and BGLAP protein secreted into conditioned media was quantified by Western blot. Mean \pm SD * is significantly different from negative control (NC) (right panel). (E) ALP staining was visualized at $10 \times$ magnification. ( F) Deposition of calcium was visualized (left panel) and quantified (right panel), by Alizarin red stain in HOBs after they were treated in osteogenic medium but for $12 \mathrm{~d}$.

\section{MATERIALS AND METHODS}

\section{Bone cell culture and transfection}

Primary human osteoblast $(\mathrm{HOB})$ cells were isolated from human trabecular bone excised from the unaffected distal region of bone specimens obtained in conjunction with prosthesis surgery. Specimens were collected from 104 donors from a cohort of patients with osteoarthritis undergoing total hip or knee replacement, with no other bone-related pathologies reported (Grundberg et al. 2009, 2011). The bone chips obtained from each donor were thoroughly minced and washed with PBS. The minced bone 
A

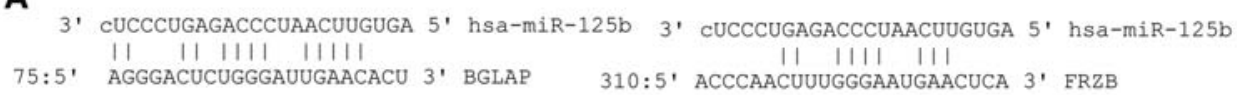

B

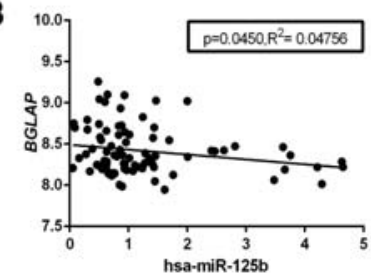

C

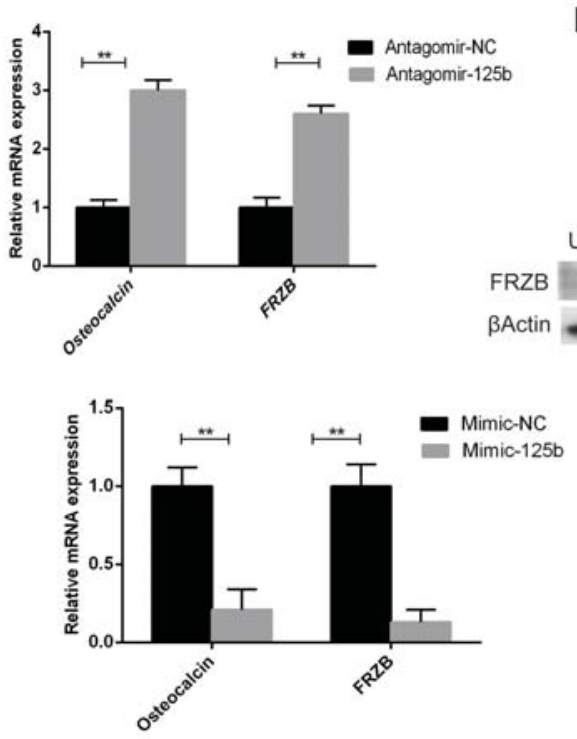

D

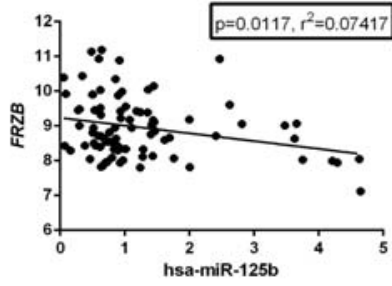

$\mathbf{F}$

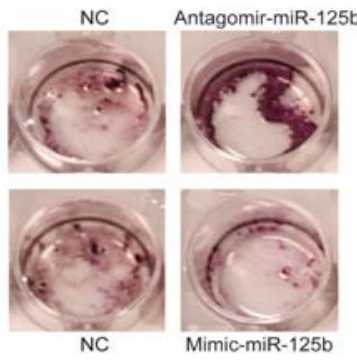

E

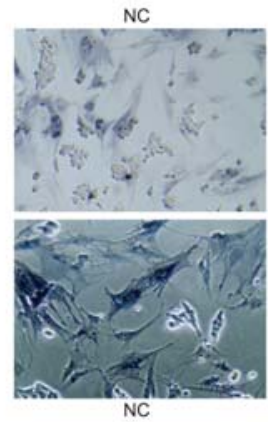

Antagomir-miR-125b
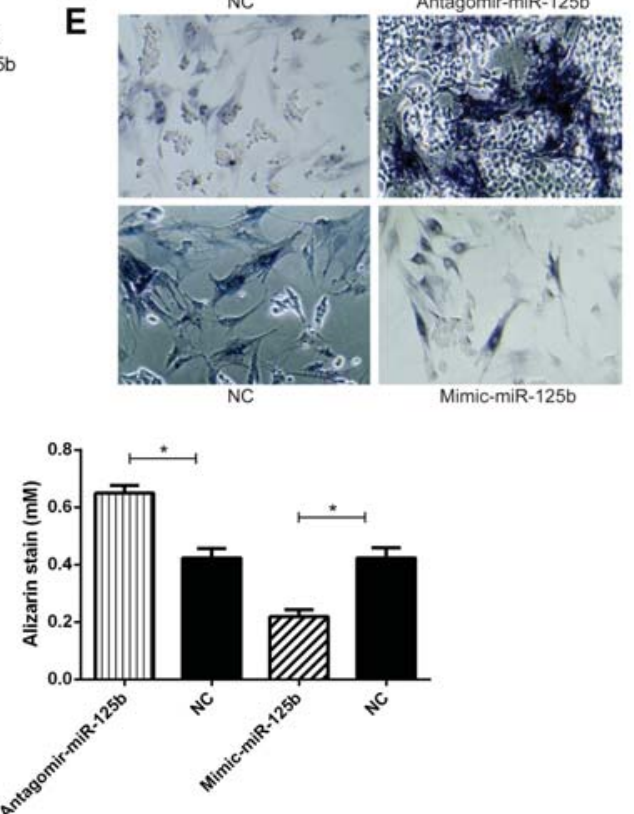

FIGURE 5. hsa-miR-125b negatively regulates BGLAP and FRZB in primary human bone cells. $(A)$ Predicted binding sites for hsa-miR-125b in BGLAP and FRZB 3' UTR by the TargetScan, miRanda, and PicTar softwares. (B) Pearson correlation scatter plots showing a negative correlation between hsa-miR-125b and BGLAP $(P=0.0450)$ and FRZB $(P=0.0117)$. (C) RT-PCR analysis of BGLAP and FRZB mRNA levels normalized to GAPDH. (D) The amount of FRZB protein was assessed by Western blot analysis with $\beta$-actin as a loading control (left panel) and BGLAP protein secreted into conditioned media was quantified by Western blot. Mean $\pm \mathrm{SD} *$ is significantly different from negative control (NC) (right panel). (E) ALP staining was visualized at $10 \times$ magnification. $(F)$ Deposition of calcium was visualized (left panel) and quantified (right panel), by Alizarin red stain in HOBs after they were treated in osteogenic medium but for $12 \mathrm{~d}$.

chips were cultured in medium containing a-MEM (Sigma-Aldrich) supplemented with $2 \mathrm{mmol} / \mathrm{L}$ L-glutamine, 100 units $/ \mu \mathrm{L}$ penicillin, $100 \mathrm{mg} / \mu \mathrm{L}$ streptomycin, and $10 \%$ fetal bovine serum (Sigma-Aldrich) at $37^{\circ} \mathrm{C}$ with $5 \% \mathrm{CO}_{2}$ until confluence was reached. The culture medium was changed twice weekly. The study was approved by the local ethics committee (Ethical approval \# Ups 03-561).
HOBs from seven individuals were seeded at a density of 35,000 cells from the second passage in a 24-well cell culture plate to achieve $60 \%-80 \%$ confluence at the day of transfection. To over express and inhibit the function of hsa-miR-29b, hsa-miR-125b, and hsa-miR30c2 the cells were transfected with mirVana hsa-miR-29b mimic (Ambion, catalog no. 4464066), mirVana hsa-miR-29b inhibitor (Ambion, catalog no. 4464084), mirVana hsa-miR-125b mimic 


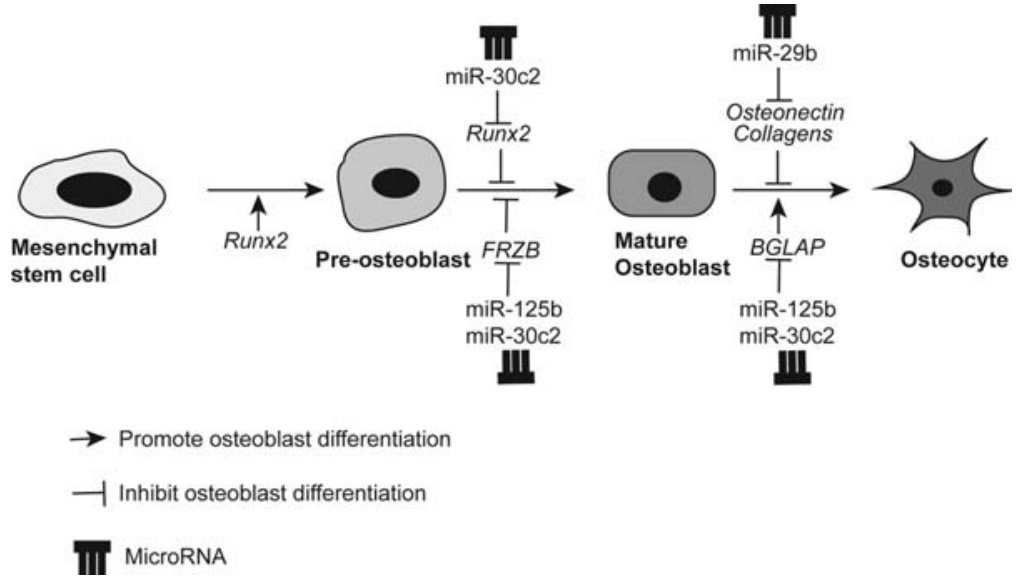

FIGURE 6. Regulation of osteoblast differentiation by miRNAs. MiRNAs influence different stages of osteoblast differentiation from mesenchymal stem cell to osteocyte. MiRNAs target important transcription factors and genes and affect regulation during each step of differentiation. MiRNAs may have a positive or negative effect depending on the target and stage of osteoblast differentiation. Runt-related transcription factor 2 (RUNX2), osteocalcin (BGLAP), and frizzled-related protein $(F R Z B)$.

(Ambion, catalog no. 4464066), mirVana hsa-miR-125b inhibitor (Ambion, catalog no. 4464084), mirVana hsa-miR-30c mimic (Ambion, catalog no. 4464066), mirVana hsa-miR-30c inhibitor (Ambion, catalog no. 4464084), and with mirVana miRNA inhibitor negative control \#1 (Ambion, catalog no. 4464077) and mirVana miRNA mimic negative control \#1 (Ambion, catalog no. 4464061) at $40 \mathrm{nM}$ concentrations with Magnet Assisted Transfection (MATra-si) reagent (IBA GmbH) according to the manufacturers' protocols. Each transfection was performed in triplicate. Post-transfection, cells were incubated for $48 \mathrm{~h}$.

\section{RNA isolation}

Total RNA was isolated from the HOB cells by adding $500 \mu \mathrm{L}$ of lysis buffer (Qiagen). The cell lysates were homogenized with QIAshredder (Qiagen, Germany). RNA was extracted from the cell lysates with the RNeasy Mini Kit (Qiagen). High RNA quality was confirmed for all samples with an Agilent 2100 BioAnalyzer (Agilent Technologies, Palo Alto, CA), where samples with RNA Integrity Number (RIN) above seven were taken forward for analysis (Schroeder et al. 2006). The concentrations were determined with NanoDrop ND-1000 (NanoDrop Technologies). For RNA in the LNA microarray pipeline, RIN values were 6.2-9.5, and OD 260/ 280 between 1.95 and 2.03. Of the original 104 samples, a total of 95 samples from 50 males and 45 females (Supplemental Fig. 1) had RIN values above threshold and were carried forward for further analyses.

\section{microRNA profiling in human bone samples by LNA microarray}

Total RNA from 11 females and 8 males were labeled with Hy3 and common reference with Hy5 fluorescent label, using the miRCURY LNA Array power labeling kit (Exiqon). The miRCURY LNA array microarray slides were scanned using the Agilent G2565BA Microarray Scanner System (Agilent Technologies, Inc.) and the image analysis was carried out using the ImaGene 8.0 software (BioDis- covery, Inc.). The quantified signals were background corrected using Normexp with offset value 10, (Ritchie et al. 2007) and normalized using the global LOESS (LOcally WEighted Scatterplot Smoothing) nonlinear regression algorithm (details provided in Supplemental Methods), whereafter data were subjected to unsupervised hierarchical clustering (Eisen et al. 1998) using MeV 4.9 release (MultiExperiment Viewer).

\section{Real-time RT-PCR}

Total RNA was reverse transcribed enabling miRNA specific cDNA synthesis for the 10 different human miRNAs and 2 miRNA controls. RT-PCR performed on a 7500 Fast RealTime PCR System and the comparative quantitation $2^{-\Delta \Delta \mathrm{CT}}$ method (Livak and Schmittgen 2001) was used to compare differences in cycle number thresholds for samples normalized for endogenous controls (details provided in Supplemental Methods).

\section{Global mRNA expression}

mRNA-expression profiling of the 95 samples, each with three biological replicates, was performed using the Illumina HumRef-8v2 BeadChips (Illumina, Inc.) where $200 \mathrm{ng}$ of total RNA was processed according to the protocol supplied by Illumina as previously reported (Grundberg et al. 2009) The biological replicates were always separated and hybridized on different BeadChips in order to avoid that results are confounded by technical effects. Any sample or array that showed an abnormal distribution of signal intensities as visualized in box plots was removed or rehybridized. The raw data were imported to Bioconductor (Gentleman et al. 2004) using the R 2.5.0 package for variance-stabilizing transformation and robust spline normalization to obtain normalized mean-expression values.

\section{Western blot analysis}

HOBs were washed twice with ice-cold PBS and total cell lysates was prepared using RIPA lysis buffer (50 mM Tris- $\mathrm{HCl}, \mathrm{pH} 8.0$, with $150 \mathrm{mM} \mathrm{NaCl}, 1.0 \%$ Igepal CA-630 (NP-40), 0.5\% sodium deoxycholate, $0.1 \%$ SDS, supplemented with $1.0 \%$ protease inhibitor cocktail (Sigma-Aldrich). Lysates were incubated on ice for 30 min and centrifuged at 10,000-RPM for 20 min to collect supernatant. For estimation of osteocalcin and osteonectin protein levels, HOBs were grown and in conditioned media (serum free) and after transfection, the medium was harvested to quantify the secreted protein, osteocalcin and osteonectin. Conditioned media was precipitated with 4 volume of cold acetone and resuspended in RIPA buffer. Protein concentration was quantified by using Coomassie Plus-The Better Bradford Assay Reagent (Thermo Scientific). Twenty micrograms of soluble protein was subjected to SDSPAGE and the separated proteins were transferred to polyvinylidene difluoride membrane (Millipore). The protein bands were probed with primary antibodies against Col1A1 (1:1000 dilution, a kind gift of Dr. Larry Fisher [NIDCR, National Institutes of Health]), 
BGLAP (1:1000 dilution, GeneTex), SPARC (ab89218, 1:1000 dilution; Abcam), FRZB (1:1000 dilution, Sigma), RUNX2 (1:250 dilution, Sigma), and ACTB (1:1000 dilution; Cell Signaling Technology). Anti-Rabbit-HRP conjugated secondary antibodies (1:3000 dilution; R\&D Systems) were used to detect the primary antibodies, followed by the target protein visualization with EMD Millipore Immobilon Western Chemiluminescent HRP Substrate (ECL). Images were acquired using LI-COR Odyssey Fc DualMode Imaging system (LI-COR Biosciences) and Image Studio Software.

\section{Data analysis}

\section{RT-PCR-expression normalization}

Assessment of the stability of the miRNA gene expression was performed using softwares geNorm, version 3.5 (Vandesompele et al. 2002) and Normfinder (Andersen et al. 2004). The program geNorm is a Visual Basic application tool for Microsoft Excel. The program selects from a panel of candidate reference genes the two most stable genes or a combination of multiple stable genes for normalization (Fu et al. 2009). The NormFinder (freely available on the Internet http://www.mdl.dk) is a Microsoft Excel add-in and calculates the stability values of the individual candidate reference genes for normalization (Vandesompele et al. 2002). A low stability value indicating a low combined intra- and inter-group variation proves high expression stability. Using this approach, the most stable single gene is calculated.

\section{Downstream data analysis}

Downstream data analysis was carried out with R statistical computing framework version 2.11.1 (http://www.R-project.org) (The R Foundation for Statistical Computing 2010) using tools from Bioconductor (Gentleman et al. 2004). The association between paired miRNA and mRNA profiles was tested by Pearson correlation coefficients. False Discovery Rate (FDR) and $P$-values $<0.05$ were also computed. The R software package adjusts $P$-values generated in multiple hypotheses testing of gene expression data. The software applies multiple testing procedures that control the False Discovery Rate (FDR) criterion introduced by Benjamini and Hochberg (1995). For visualization of miRNA and mRNA correlations, the Statistica v10 software was used (StatSoft, Inc.).

\section{Overrepresentation analysis of predicted miRNA targets}

Predictions of genes carrying conserved miRNA target sites in their UTRs were downloaded from the Targetscan website (Lewis et al. 2005). We then further analyzed hsa-miR-29b since it had the largest number of significant correlations to mRNA levels. (details provided in Supplemental Methods).

\section{Bioinformatic analysis of correlations - ingenuity} pathway analysis

The ingenuity pathway analysis (IPA; Ingenuity Systems, www. ingenuity.com), a web-delivered application, was used to explore molecular functions and relevant networks and gene targets (Mori et al. 2009), where the significant correlations were associated and/ or overrepresented (details provided in Supplemental Methods).

\section{Statistical analysis}

Correlations between miRNA fold-change values and mRNA-expression values were determined using the $\mathrm{R}$ statistical software 3.11.1 package in Bioconductor (Release 3.0). The Student's $t$-test was used to determine statistical differences between pairs of groups. Two-way analysis of variance (ANOVA) was used to evaluate the statistical significance for comparisons within groups. $P<0.05$ (two sided) was considered as statistically significant. $P$-values obtained were adjusted for false discovery rate due to multiple testing correction (Benjamini and Hochberg 1995). For generation of correlation graphs and statistical analysis, Statistica v12 software (StatSoft, Inc.) and GraphPad Prism software package (version $6.0)$ were used.

\section{SUPPLEMENTAL MATERIAL}

Supplemental material is available for this article.

\section{ACKNOWLEDGMENTS}

This work was supported by Swedish Research Council, ALF-grants, and Torsten \& Ragnar Söderberg Foundation.

Received January 7, 2015; accepted April 21, 2015.

\section{REFERENCES}

Andersen CL, Jensen JL, Ørntoft TF. 2004. Normalization of real-time quantitative reverse transcription-PCR data: a model-based variance estimation approach to identify genes suited for normalization, applied to bladder and colon cancer data sets. Cancer Res 64: 5245-5250.

Baglìo SR, Devescovi V, Granchi D, Baldini N. 2013. MicroRNA expression profiling of human bone marrow mesenchymal stem cells during osteogenic differentiation reveals Osterix regulation by miR-31. Gene 527: 321-331.

Bartel DP. 2004. MicroRNAs: genomics, biogenesis, mechanism, and function. Cell 116: 281-297.

Benjamini Y, Hochberg Y. 1995. Controlling the false discovery rate: a practical and powerful approach to multiple testing. J $R$ Stat Soc Ser B Methodol 57: 289-300.

Davis BN, Hata A. 2009. Regulation of microRNA biogenesis: a miRiad of mechanisms. Cell Commun Signal 7: 18.

Doench JG, Sharp PA. 2004. Specificity of microRNA target selection in translational repression. Genes Dev 18: 504-511.

Eisen MB, Spellman PT, Brown PO, Botstein D. 1998. Cluster analysis and display of genome-wide expression patterns. Proc Natl Acad Sci 95: 14863-14868.

Engels BM, Hutvagner G. 2006. Principles and effects of microRNAmediated post-transcriptional gene regulation. Oncogene 25: 61636169.

Fu LY, Jia HL, Dong QZ, Wu JC, Zhao Y, Zhou HJ, Ren N, Ye QH, Qin LX. 2009. Suitable reference genes for real-time PCR in human HBV-related hepatocellular carcinoma with different clinical prognoses. BMC Cancer 9: 49.

Gao J, Yang T, Han J, Yan K, Qiu X, Zhou Y, Fan Q, Ma B. 2011. MicroRNA expression during osteogenic differentiation of human multipotent mesenchymal stromal cells from bone marrow. J Cell Biochem 112: 1844-1856.

Gentleman RC, Carey VJ, Bates DM, Bolstad B, Dettling M, Dudoit S, Ellis B, Gautier L, Ge Y, Gentry J, et al. 2004. Bioconductor: open software development for computational biology and bioinformatics. Genome Biol 5: R80. 
Grundberg E, Kwan T, Ge B, Lam KC, Koka V, Kindmark A, Mallmin H, Dias J, Verlaan DJ, Ouimet M, et al. 2009. Population genomics in a disease targeted primary cell model. Genome Res 19: 1942-1952.

Grundberg E, Adoue V, Kwan T, Ge B, Duan QL, Lam KC, Koka V, Kindmark A, Weiss ST, Tantisira K, et al. 2011. Global analysis of the impact of environmental perturbation on cis-regulation of gene expression. PLoS Genet 7: e1001279.

Guo H, Ingolia NT, Weissman JS, Bartel DP. 2010. Mammalian microRNAs predominantly act to decrease target mRNA levels. Nature 466: 835-840.

$\mathrm{Hu}$ W, Coller J. 2012. What comes first: translational repression or mRNA degradation? The deepening mystery of microRNA function. Cell Res 22: 1322-1324.

Inui M, Martello G, Piccolo S. 2010. MicroRNA control of signal transduction. Nat Rev Mol Cell Biol 11: 252-263.

Kalajzic I, Staal A, Yang WP, Wu Y, Johnson SE, Feyen JH, Krueger W, Maye P, Yu F, Zhao Y, et al. 2005. Expression profile of osteoblast lineage at defined stages of differentiation. J Biol Chem 280: 24618-24626.

Kapinas K, Kessler CB, Delany AM. 2009. miR-29 suppression of osteonectin in osteoblasts: regulation during differentiation and by canonical Wnt signaling. J Cell Biochem 108: 216-224.

Kapinas K, Kessler C, Ricks T, Gronowicz G, Delany AM. 2010. miR-29 modulates Wnt signaling in human osteoblasts through a positive feedback loop. J Biol Chem 285: 25221-25231.

Lewis BP, Burge CB, Bartel DP. 2005. Conserved seed pairing, often flanked by adenosines, indicates that thousands of human genes are microRNA targets. Cell 120: 15-20.

Li Z, Hassan MQ, Jafferji M, Aqeilan RI, Garzon R, Croce CM, van Wijnen AJ, Stein JL, Stein GS, Lian JB. 2009. Biological functions of miR-29b contribute to positive regulation of osteoblast differentiation. J Biol Chem 284: 15676-15684.

Li ZI, Chung AC, Zhou L, Huang XR, Liu F, Fu P, Fan JM, Szalai AJ, Lan HY. 2011. C-reactive protein promotes acute renal inflammation and fibrosis in unilateral ureteral obstructive nephropathy in mice. Lab Invest 91: 837-851.

Lian JB, Stein GS, van Wijnen AJ, Stein JL, Hassan MQ, Gaur T, Zhang Y. 2012. MicroRNA control of bone formation and homeostasis. Nat Rev Endocrinol 8: 212-227.

Livak KJ, Schmittgen TD. 2001. Analysis of relative gene expression data using real-time quantitative PCR and the $2(-\Delta \Delta C(\mathrm{~T}))$ method. Methods 25: 402-408.

Martinez NJ, Ow MC, Barrasa MI, Hammell M, Sequerra R, DoucetteStamm L, Roth FP, Ambros VR, Walhout AJ. 2008. A C. elegans genome-scale microRNA network contains composite feedback motifs with high flux capacity. Genes Dev 22: 2535-2549.

Miyazono K, Maeda S, Imamura T. 2005. BMP receptor signaling: transcriptional targets, regulation of signals, and signaling cross-talk. Cytokine Growth Factor Rev 16: 251-263.

Mori R, Xiong S, Wang Q, Tarabolous C, Shimada H, Panteris E, Danenberg KD, Danenberg PV, Pinski JK. 2009. Gene profiling and pathway analysis of neuroendocrine transdifferentiated prostate cancer cells. Prostate 69: 12-23.

Nunez-Iglesias J, Liu CC, Morgan TE, Finch CE, Zhou XJ. 2010. Joint genome-wide profiling of miRNA and mRNA expression in Alzheimer's disease cortex reveals altered miRNA regulation. PloS One 5: e8898.

Philippidou D, Schmitt M, Moser D, Margue C, Nazarov PV, Muller A, Vallar L, Nashan D, Behrmann I, Kreis S. 2010. Signatures of microRNAs and selected microRNA target genes in human melanoma. Cancer Res 70: 4163-4173.

Rajewsky N. 2006. microRNA target predictions in animals. Nat Genet 38 Suppl: S8-S13.

Ritchie ME, Silver J, Oshlack A, Holmes M, Diyagama D, Holloway A, Smyth GK. 2007. A comparison of background correction methods for two-colour microarrays. Bioinformatics 23: 2700-2707.

Schroeder A, Mueller O, Stocker S, Salowsky R, Leiber M, Gassmann M, Lightfoot S, Menzel W, Granzow M, Ragg T. 2006. The RIN: an RNA integrity number for assigning integrity values to RNA measurements. BMC Mol Biol 7: 3 .

Seeliger C, Karpinski K, Haug A, Vester H, Schmitt A, Bauer J, van Griensven M. 2014. Five freely circulating miRNAs and bone tissue miRNAs are associated with osteoporotic fractures. J Bone Miner Res 29: $1718-1728$.

Sun J, Gong X, Purow B, Zhao Z. 2012. Uncovering microRNA and transcription factor mediated regulatory networks in glioblastoma. PLoS Comput Biol 8: e1002488.

Vandesompele J, De Preter K, Pattyn F, Poppe B, Van Roy N, De Paepe A, Speleman F. 2002. Accurate normalization of real-time quantitative RT-PCR data by geometric averaging of multiple internal control genes. Genome Biol 3: RESEARCH0034.

Vasudevan S, Tong Y, Steitz JA. 2007. Switching from repression to activation: microRNAs can up-regulate translation. Science 318: 1931-1934.

Vergoulis T, Vlachos IS, Alexiou P, Georgakilas G, Maragkakis M, Reczko M, Gerangelos S, Koziris N, Dalamagas T, Hatzigeorgiou AG. 2012. TarBase 6.0: capturing the exponential growth of miRNA targets with experimental support. Nucleic Acids Res 40: D222-D229.

Wilczynska A, Bushell M. 2015. The complexity of miRNA-mediated repression. Cell Death Differ 22: 22-33.

Zhang Y, Xie RL, Croce CM, Stein JL, Lian JB, van Wijnen AJ, Stein GS. 2011. A program of microRNAs controls osteogenic lineage progression by targeting transcription factor Runx2. Proc Natl Acad Sci 108: 9863-9868.

Zhao X, Xu D, Li Y, Zhang J, Liu T, Ji Y, Wang J, Zhou G, Xie X. 2014. MicroRNAs regulate bone metabolism. J Bone Miner Metab 32: 221-231.

Zhou L, Liu F, Huang XR, Liu F, Chen H, Chung AC, Shi J, Wei L, Lan HY, Fu P. 2011. Amelioration of albuminuria in ROCK1 knockout mice with streptozotocin-induced diabetic kidney disease. Am J Nephrol 34: 468-475. 

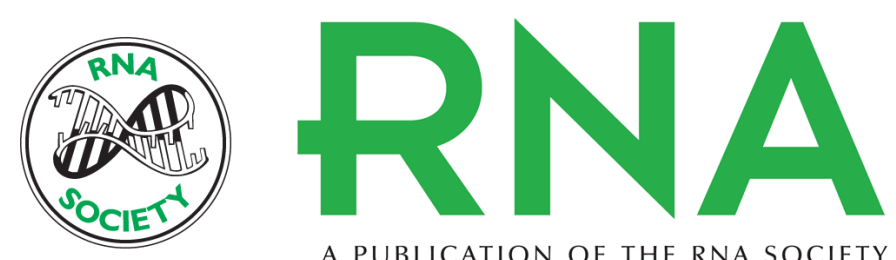

A PUBLICATION OF THE RNA SOCIETY

\section{Global miRNA expression and correlation with mRNA levels in primary human bone cells}

Navya Laxman, Carl-Johan Rubin, Hans Mallmin, et al.

RNA 2015 21: 1433-1443 originally published online June 15, 2015

Access the most recent version at doi:10.1261/rna.049148.114

\section{Supplemental http://rnajournal.cshlp.org/content/suppl/2015/06/02/rna.049148.114.DC1 \\ Material}

References This article cites 41 articles, 11 of which can be accessed free at: http://rnajournal.cshlp.org/content/21/8/1433.full.html\#ref-list-1

Open Access Freely available online through the RNA Open Access option.

Creative This article, published in $R N A$, is available under a Creative Commons License Commons (Attribution-NonCommercial 4.0 International), as described at License http://creativecommons.org/licenses/by-nc/4.0/.

Email Alerting Receive free email alerts when new articles cite this article - sign up in the box at the Service top right corner of the article or click here. 